\title{
Four Dimensional MR Image Analysis of Dynamic Renography
}

\author{
Ting Song, Vivian S. Lee, Henry Rusinek, Samson Wong, and Andrew F. Laine
}

\begin{abstract}
A novel four dimensional image analysis approach including registration and segmentation of dynamic contrast enhanced renal MR images is presented. This integrated method is motivated by the observation of the reciprocity between registration and segmentation in 4D time-series images. Fully automated Fourier-based registration with sub-voxel accuracy and semi-automated time-series segmentation were intertwined to improve the accuracy in a multi-step fashion. We have tested our algorithm on several real patient data sets. Clinical validation showed remarkable and consistent agreement between the proposed method and manual segmentation by experts.
\end{abstract}

\section{INTRODUCTION}

$\mathrm{F}$ OUR dimensional dynamic contrast enhanced renal magnetic resonance (MR) imaging is increasely used to assess renal functions. This noninvasive and safe procedure is based on renal T1-weighted imaging acquired during 5-10 minutes after intravenous injection of a low dose of gadopentetate dimeglumine. For each kidney, the signal intensity can be measured in intrarenal compartments (cortex, medulla, and collecting system) and combined with arterial input function, used to compute the renal blood flow, blood volume, single kidney glomerular filtration rate (GFR) and other functional parameters in vivo [1]. Dynamic MR renography has broad clinical applications, but it requires an extensive image analysis that is complicated by respiratory motion. Since each examination yields at least 10-20 serial $3 \mathrm{D}$ images of the abdomen, manual registration and segmentation are prohibitively labor-intensive. Therefore, automated and semi-automated image registration and segmentation techniques to analyze dynamic MR renography are of great clinical interest.

\section{A. Kidney Segmentation}

The challenging part for dynamic contrast enhanced image segmentation is that when contrast agent wash-in and wash-out ocurrs, image intensity values change rapidly as the time series evolves. Of course poor kidney function or stenotic vasculaturemay prevent the uptake of contrast agent, resulting in disjoined bright regions. Accurate and continuous boundary delineation is not always feasible. Moreover, the

Manuscript received April 20 $0^{\text {th }}, 2006$.

Ting Song and Andrew Laine are with the Department of Biomedical Engineering, Columbia University, New York, NY 10027 USA. (Phone: 212-854-5996; fax: 212-854-5995; e-mail: ts2060@ Columbia.edu and laine@columbia.edu).

Vivian S. Lee, Henry Rusinek, and Samson Wong are with the Department of Radiology, New York University, New York, NY 10016 USA. (E-mail:Vivian.Lee@med.nyu.edu, hr18@nyu.edu, and sw345@med.nyu.edu). contrast agent can also wash into neighbor tissues, such as the spleen and liver.

Basically, kidney segmentation techniques can be divided in two basic categories: spatial or temporal. In the spatial approach segmentation is performed separately at each time point $[2,3]$. Temporal or vector segmentation considers each voxel's intensity time course as a vector and classifies the tissues according their different features and behaviors occurring in the temporal domain [4-7].

Because the passage of contrast needs to be observed for several minutes over multiple breath-holds, dynamic imaging is affected by respiratory motion. Clearly the accuracy of kidney segmentation will strongly depend on robust registration over time.

\section{B. Kidney Registration}

There has been limited work related to the registration of dynamic MRI data applicable to the abdominal region. These methods include mutual information (MI) [5], contour and template matching based methods [7-10], and phase correlation [11]. Prior work was restricted to either in-plane 2D motion or 3D translation only. The proposed automated method is more general and it corrects for both rotation and translation motion in $3 \mathrm{D}$.

Without kidney contour segmentation, time series image registration is challenging. Therefore, for dynamic perfusion images, segmentation and registration presents the well-known dilemma of "chicken and egg". Next we show that when the two methods are integrated and implemented alternatively, the overall performance is improved.

\section{Methodology}

With this synergy in mind, a computer-aided integrated method was developed to cross thread image registration and segmentation processing of dynamic 3D MR renography. The flowchart of our method is shown in Fig. 1. The diagram shows the specific modules of interlacing registration and segmentation procedures used to improve overall performance of the integrated system. Similar ideas can be found in [7], where rough registration, segmentation, and fine registration were performed respectively. 


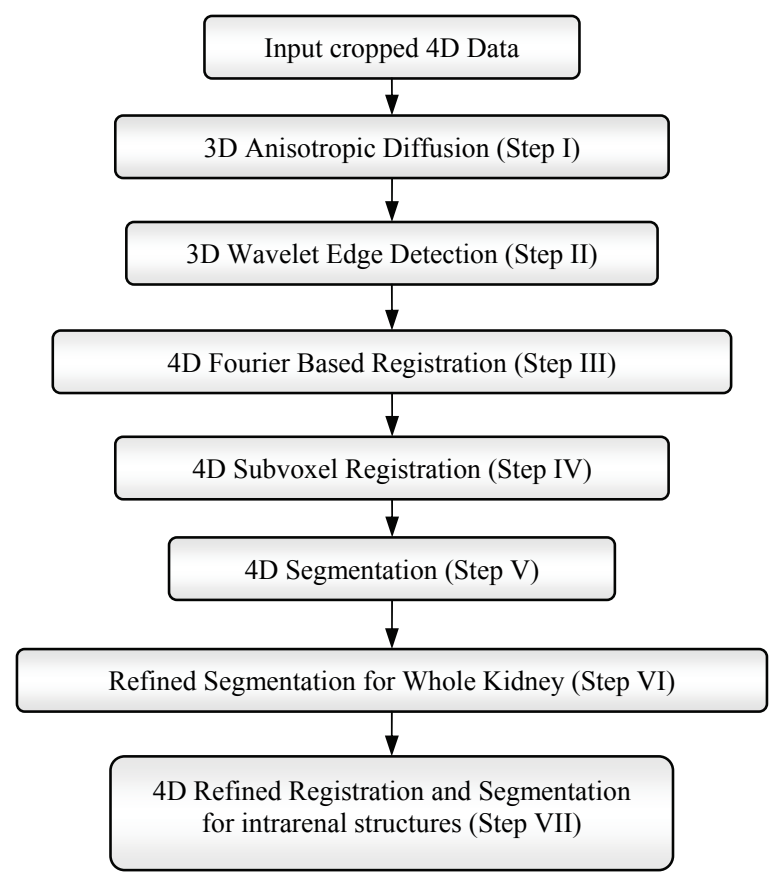

Fig. 1.Flowchart of proposed integrated algorithm showing intertwining of processing modules.

\section{A. Preprocessing and Rough Registration (Steps I-IV)}

Given a 4D MR renography data set, after cropping kidney out of the whole body images, 3D anisotropic diffusion with time-varying gradient threshold was applied to suppress noise as well as preserve image features. Then, a 3D over-complete dyadic wavelet expansion was applied and the modulus of wavelet coefficients from the detail channel at the $2^{\text {nd }}$ level served as robust edge information. These representations were fed into the next step, rough $4 \mathrm{D}$ registration. The rough 4D registration utilized a Fourier-based method to provide a robust estimation with voxel-wise accuracy for translation and rotation between each frame. Rotation and translation processing were separated by the Fourier Transform. The rotation between each frame was recovered by minimizing following energy function

$$
E=\iiint\left(\mathfrak{F}[g]\left(w_{x}, w_{y}, w_{z}\right)-\mathfrak{F}[R f]\left(w_{x}, w_{y}, w_{z}\right)\right)^{2} d w_{x} d w_{y} d w_{z},
$$

and the optimum rotation axis and rotation angle was recovered by

$$
(\hat{\theta}, \hat{\phi}, \hat{\psi})=\underset{\theta, \phi, \psi}{\arg \min } E
$$

The minimization problem in equation (1) can be efficiently solved by the Quasi-Newton Method [12]. This was the first time that $3 \mathrm{D}$ rotation issue was addressed in $4 \mathrm{D}$ MR renography image processing techniques.

Next, the translation vector was estimated by a phase-correlation technique

$$
\widehat{\operatorname{corr}}=\frac{\mathfrak{F}[g]\left(w_{x}, w_{y}, w_{z}\right) \mathfrak{F}^{*}[R f]\left(w_{x}, w_{y}, w_{z}\right)}{\left|\mathfrak{F}[g]\left(w_{x}, w_{y}, w_{z}\right) \| \mathfrak{F}[R f]\left(w_{x}, w_{y}, w_{z}\right)\right|}=e^{j\left[w_{x} w_{y} w_{z}\right] \cdot t},
$$

where $R$ stands for the rotation matrix and $\mathfrak{F}$ stands for 3D Fourier Transform.

To improve the registration accuracy, a 3D subvoxel registration method, extended from the work of Stone, et al [13] to three-dimensional gray-scale images in [14], was applied. By using phase properties of the Fourier transform, estimation for sub-voxel registration can be modeled as a classical linear fitting problem of the following system:

$$
\frac{2 \pi}{N}\left[\begin{array}{lll}
w_{x} & w_{y} & w_{z}
\end{array}\right]\left[\begin{array}{lll}
x_{0} & y_{0} & z_{0}
\end{array}\right]^{T}=[\operatorname{phase}(\widetilde{F} / \widetilde{G})],
$$

which can be solved by pseudo inverse methods or singular value decomposition for the subvoxel translation vector $\left[x_{0}, y_{0}, z_{0}\right]^{T}$. For details of the preprocessing and rough registration steps, please refer to our previous work [14].

\section{B. 4D Time-Series Segmentation (Step V)}

The temporal pattern of contrast uptake is a strong feature to distinguish kidney tissues from surrounding tissues as well as to differentiate different intrarenal tissues. Due to differences invasculature, filtration, and reabsorption, different tissue of interest enhance at different phases of the acquisition. For example, in a normal kidney, the peak uptake in the cortex occurs about 30 seconds after injection, in the medulla at 2-3 minutes, and in the collecting system at 4-6 minutes. In order to utilize the temporal information, a normative template pattern was constructed for the background and three renal tissues (cortex, medulla, collecting systems). Then the mean temporal dynamics for each class was derived for further temporal resolution. Thus the time-series segmentation algorithm is based on minimization of the following energy functional

$$
\sum_{n=1}^{N} \sum_{v \in C_{n}} \operatorname{dis}\left(\left|T D(v)-\overline{T D_{C_{n}}}\right|\right),
$$

where $\overline{T D_{C_{n}}}$ denotes the normative temporal dynamic pattern of tissue $C_{n}$. In equation (5), $v$ presents for a voxel belonging to class $C_{n}$. The function dis stands for the Euclidean distance in T-dimensional vector space, where $\mathrm{T}$ is the total number of frames in each $4 \mathrm{D}$ series; $T D(v)$ stands for the temporal dynamics for voxel $v$. After this step, rough segmentation for each tissue of interest was generated.

\section{Refined Segmentation for Whole Kidney Volume (Step $V I)$}

In order to reduce errors resulting from initial registration and segmentation, additional registration and segmentation steps are applied. The initial mask for the whole kidney was generated by combining the segmentation results for the cortex, medulla, and collecting system from the previous step. A narrowband mask containing voxels apart from the initial boundary of the whole kidney within \pm 3 voxels distance range was generated for each frame. Then, within this mask, a refined kidney boundary for each frame was segmented using the methods described in [15]. 


\section{4D Refined Registration and Segmentation for Intrarenal Structures (Step VII)}

After the segmentations for the kidney boundaries were refined for each frame in the previous step, the binary images series of the kidney shell was used for calculating the refined parameters for registration, i.e. translation and rotation, using the binary version of the Fourier-based registration method with sub-voxel accuracy proposed in [14]. After the image series was realigned using the refined registration step, an additional temporal segmentation similar to the method used in Step V was applied to segment the intrarenal tissues of the kidney.

\section{E. Clinical Evaluation}

The above procedure was first tested on 16 pairs (object image and target image) of kidney contours (four subjects, four pairs of volumes) that represented small, medium and large $(<1 \mathrm{~mm}, 1-5 \mathrm{~mm}$, and $>5 \mathrm{~mm})$ degrees of kidney motion. The images were selected from MR examinations of four subjects with renal insufficiency. An expert observer manually traced kidney contours to provide registration ground truth to evaluate the performance of the automated registration. Coordinates were defined as: $\mathrm{x}=$ head to feet, $\mathrm{y}=$ left to right, $\mathrm{z}=$ anterior to posterior). Conventional rotation angle parameters were expressed in degrees $(\theta, \varphi, \psi)$.

The proposed method was then evaluated on $4 \mathrm{D}$ MR renography data sets from four patients with 41 time phases per kidney, with manual segmentation and registration performed by two M.D. experts in body radiology. Manual segmentations of the cortex, medulla and collecting system were used as the reference standard to identify segmentation overlap and volume evaluation. All translation results were expressed in voxels and an absolute voxel size $(1.66,1.66$, 2.5) $\mathrm{mm}$ was used throughout the study.

\section{EXPERIMENTS AND RESULTS}

Fig. 2 shows coregistration errors derived from 16 pairs of images. The mean value of course translation errors were $[0.5344,0.6390,0.1508]$ voxels; the mean value of refined translation errors were $[0.2416,0.5540,0.1292]$ voxels. Rotation errors were mainly from the third parameter $\psi$, which is the rotation angle. Averaged rotation errors were $[0.0000,0.0003,-0.6630]$ degrees, which represents rotation in the sagittal plane. It was also shown that the refinement in registration improved the accuracy in the final alignment.
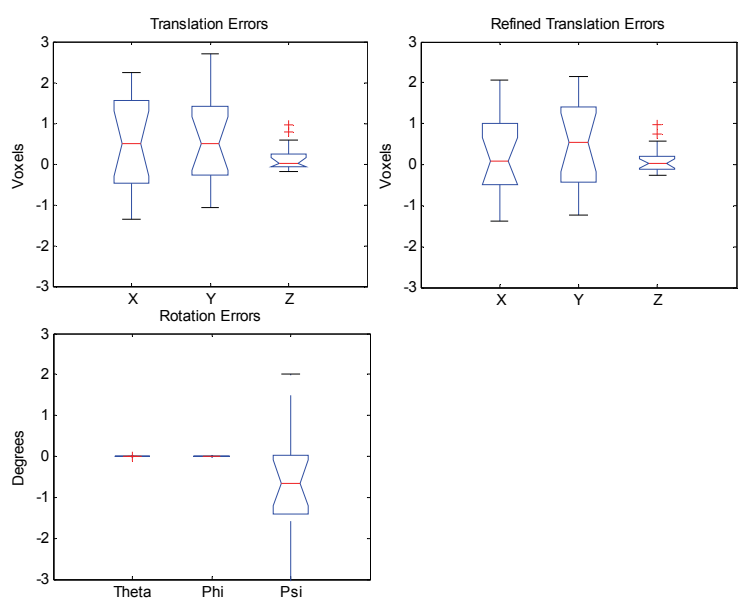

Fig. 2. Distribution of errors in 3D translation and rotation.

On the four patient data sets, segmented volumes of intrarenal regions were calculated and compared with the experts' results shown in Fig. 3. Since the computer-based method excluded the dark boundaries around kidney from the cortex whereas experts tended to include them, our method yielded consistent under-segmentation compared to manual segmentation for the cortex; for the medulla and collecting system, our method was comparable to manual segmentation. Computer aided segmentation results and experts' segmentation are illustrated at different time frames in Fig. 4. A 3D visualization of the registration results based on manually segmented ROIs and computer calculated results are also shown in Fig. 5. to illustrate the misalignment before registration and alignment after registration using manual segmentation or the computer-based method.

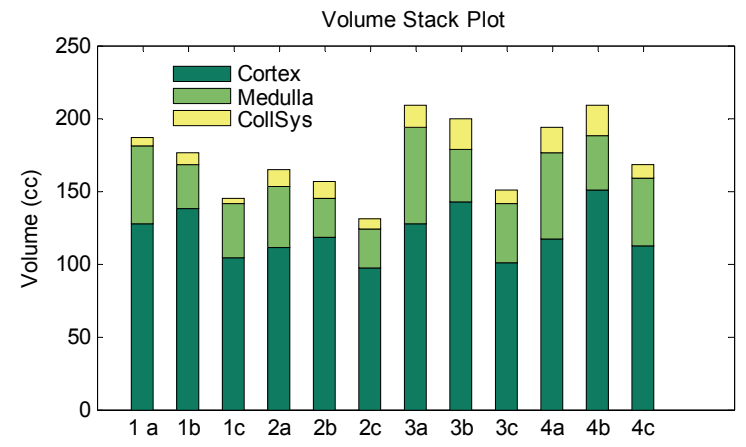

Fig. 3. Volumes of cortex, medulla and collecting system on four patient's data from 1 to 4. (a) and (b) are volumes from our two experts, and (c) are volumes from our automated method. 


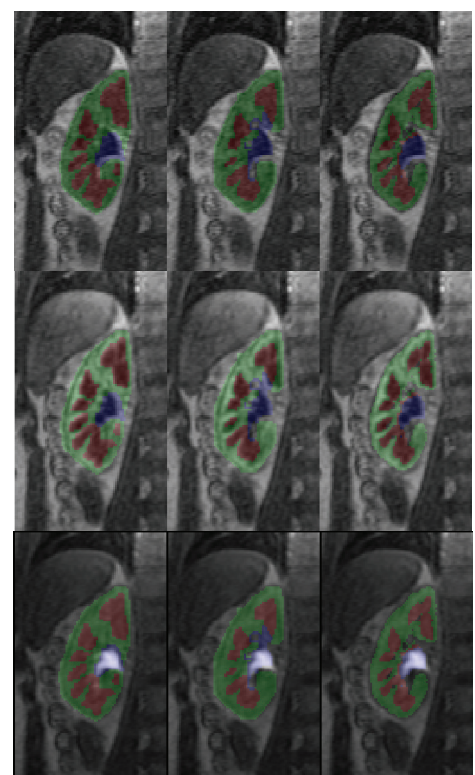

(a)

(b)

(c)

Fig. 4. Segmentation results for three different time points $1 \sim 3$ (1st row $\sim 3$ rd row) on one patient. (a) and (b) are segmented ROIs from two experts; (c) is the final segmentation from proposed algorithm, which shows great agreement with manual results. Green is cortex; red is medulla; blue is collecting system. Color labels are transparently displayed over the original gray images.

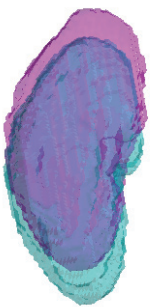

(a)

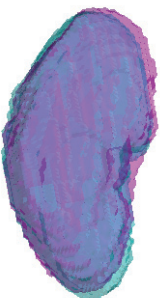

(b)

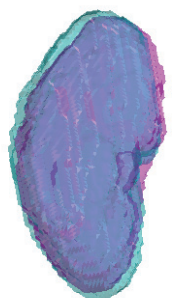

(c)
Fig. 5. Illustration of typical registration results for two different time points: (a) original position (b) registered image using manual binary ROIs, and (c) registered images using our algorithm on gray-level images.

\section{CONCLUSIONS}

In 4D (3D plus time) MRI renography, manual alignments and delineation for each 4D dataset usually requires approximately 3-4 hours of a radiologist time at a workstation per case. This remains prohibitively costly and labor-intensive for practical clinical use. In this paper, we proposed a novel four-dimensional MRI renography registration-segmentation framework. The strength of the algorithm is in the integration of image segmentation and registration, which improved the overall performance of analysis. The proposed method was quantitatively evaluated on several real patient data sets, which yielded accurate and robust results. Refined average translation errors were almost less than half of a voxel; averaged rotation error was within one degree. Therefore, the proposed image registration and segmentation algorithm appear suitable for automated analysis of clinical MR renography data.

\section{REFERENCES}

[1] V. S. Lee, H. Rusinek, M. E. Noz, P. Lee, M. Raghavan, and E. L. Kramer, "Dynamic Three-dimensional MR Renography for the Measurement of Single Kidney Function: Initial Experience," Radiology, vol. 227, pp. 289-294, 2003.

[2] S. E. Yuksel, A. El-Baz, A.A.Farag, M. E. A. El-Ghar, T.A.Eldiasty, and M.A.Ghoneim, "Automatic detection of renal rejection after kidney transplantation," International Congress Series vol. 1281, pp. 773-778, 2005.

[3] J. D. Grattan-Smith, M. R. Perez-Bayfield, R. A. Jones, S. Little, B. Broecker, E. A. Smith, H. C. Scherz, and A. J. Kirsch, "MR imaging of kidneys functional evaluation using F-15 perfusion imaging," Pediatric Radiology vol. 33, pp. 293-304, 2003.

[4] Y. Sun, J. M. F. Moura, D. Yang, Q. Ye, and C. Ho, "Kidney segmentation in MRI sequences using temporal dynamics," presented at IEEE International Symposium on Biomedical Imaging, 2002.

[5] Y. Boykov, V. S. Lee, H. Rusinek, and R. Bansal, "Segmentation of dynamic N-D data sets via graph cuts using markov models," presented at Proceedings of the 4th International Conference on Medical Image Computing and Computer-Assisted Intervention, 2001

[6] J. A. d. Priester, A. G. Kessels, E. L. Giele, J. A. d. Boer, M. H. Christiaans, A. Hasman, and J. M. v. Engelshoven, "MR renography by semiautomated image analysis: performance in renal transplant recipients," J Magn Reson Imag, vol. 14, pp. 134-140, 2001.

[7] Y. Sun, M.-P. Jolly, and J. M. F. Moura, "Integrated Registration of Dynamic Renal Perfusion MR Images," presented at IEEE International Symposium on Image Processing, ICIP'04, Singapore, 2004.

[8] Y. Sun, J. M. F. Moura, and C. Ho, "Subpixel Registration in Renal Perfusion MR Image Sequence," presented at IEEE International Symposium on BioImaging, ISBI'04, Crystal City, VA, 2004.

[9] G. Gerig, R. kikinis, W. Kuoni, G. K. v. Schulthess, and O. Kubler, "Semiautomated ROI Analysis in Dynamic MRI-Studies: PartI: Image Analysis Tools for Automatic Correction of Organ Displacement," IEEE Transaction on Image Processing, vol. 11, pp. 221-232, 1992.

[10] P. J. Yim, H. B. Marcos, M. McAuliffe, D. McGarry, and I. Heaton, "Registration of Time-Series Contrast Enhanced Magnetic Resonance Images for Renography," presented at 14th IEEE Symposium Computer-Based Medical Systems, 2001.

[11] E. L. W. Giele, J. A. dePriester, J. A. Blom, J. A. d. Boer, J. M. S. v. Engelshoven, A. Hasman, and M. Geerlings, "Movement Correction of the Kidney in Dynamic MRI Scans Using FFT Phase Difference Movement Detection," Journal of Magnetic Resonance Imaging, vol. 14, pp. 741-749, 2001.

[12] D. F. Shanno, "Conditioning of Quasi-Newton Methods for Function Minimization," Matheamtics of Computing, vol. 24, pp. 647-656, 1970.

[13] H. S. Stone, M. T. Orchard, E.-C. Chang, and S. A. Martucci, "A Fast Direct Fourier-Based Algorithm for Subpixel Registration of Images," IEEE Transaction on Geoscience and Remote Sensing, vol. 39, pp. 2235-2243, 2001.

[14] T. Song, V. S. Lee, H. Rusinek, M. Kaur, and A. F. Laine, "Automatic 4-D Registration in Dynamic MR Renography Based on Over-complete Dyadic Wavelet and Fourier Transforms," Lecture Notes in Computer Science, vol. 3749, pp. 205-213, 2005.

[15] C. Xu and J. Prince, "Snakes, Shapes, and Gradient Vector Flow," IEEE Transactions on Image Processing, vol. 7, pp. 359-369, 1998. 